\title{
Long term azithromycin therapy in patients with cystic fibrosis
}

\author{
Nagehan Emiralioğlu${ }^{1}$, Zeynelabidin Öztürk ${ }^{2}$, Ebru Yalçınn ${ }^{1}$, Deniz Doğru1 ${ }^{1}$, Uğur Özçelik ${ }^{1}$ \\ Nural Kiper ${ }^{1}$ \\ ${ }^{1}$ Division of Pediatric Pulmonology, ${ }^{2}$ Department of Pediatrics, Hacettepe University Faculty of Medicine, Ankara, Turkey. \\ Email: ebruy@hacettepe.edu.tr \\ Received: 15 March 2016, Revised: 28 April 2016, Accepted: 6 May 2016
}

SUMMARY: Emiralioğlu N, Öztürk Z, Yalçın E, Doğru D, Özçelik U, Kiper N. Long term azithromycin therapy in patients with cystic fibrosis. Turk J Pediatr 2016; 58: 34-40.

Inflammation is a central contributor to the pathogenesis of cystic fibrosis (CF) pulmonary disease; so limiting the excessive production of inflammatory mediators represents a major therapeutic strategy for slowing the decline in lung function and improving survival. The macrolide antibiotic azithromycin $(\mathrm{AZM})$ has anti-inflammatory properties and immunomodulatory effects that may be beneficial in CF. The aim of this study was to document the long term use of AZM effect on pulmonary function, nutritional status and number of pulmonary exacerbations in patients with CF.

Twenty four patients with CF aged 4-23 years followed at Hacettepe University Department of Pediatric Pulmonology between May 2007- December 2014 enrolled in the study from $630 \mathrm{CF}$ patients. They received $10 \mathrm{mg} / \mathrm{kg} / \mathrm{day}$ of AZM three times a week. Pulmonary function parameters, sputum cultures, body mass index (BMI) $\mathrm{Z}$ scores and number of pulmonary exacerbations were analyzed at different time intervals (at the visits at months 6, 9 and 12).

Median age of the patients was 14.7 (range 4-23 years) years and median treatment duration was 14 months (range 6-60 months). Initially, median FEV $1 \%$ was found $68 \%$ (range $30 \%-100 \%$ ), BMI was found 17.05 (range 13.3 26.5 ) and oxygen saturation was found $95 \%$ (range $84 \%-99 \%$ ). At the end of the 6th, 9th and 12th months of the AZM therapy; no significant differences in FEV $1 \%$ and oxygen saturation parameters were found compared to the initial time, however BMI increased significantly $(p=0.03)$, also the number of pulmonary exacerbations $(p<0.001)$ and severe exacerbations $(p<0.001)$ needing intravenous antibiotic treatment were significantly reduced at the 6 th and 12th month. At the end of the 12th month of AZM; Methicillin sensitive $S$. aureus (MSSA) colonization was significantly increased $(p=0.005)$ and increased macrolide resistance was detected $(p=0.008)$.

Although, this study could not be designed as a placebo controlled study, the results showed that at least 6 months of AZM treatment led to a significiant reduction in the number of pulmonary exacerbations requiring antibiotics and improvement on nutritional status. Despite increased $P$. aeruginosa antibiotic resistance and MSSA colonization rates, the lower incidence of acute exacerbations in patients receiving AZM is an important and clinically relevant measure of beneficial effect. Therefore, long term use of AZM may be considered to slow pulmonary deterioration in CF patients with $P$. aeruginosa colonization.

Key words: cystic fibrosis, inflammation, macrolide, lung function, nutrition. 
Cystic fibrosis (CF) is an autosomal recessive disease caused by mutations in the cystic fibrosis transmembrane conductance regulator (CFTR) gene that encodes for a protein that regulates liquid volume on epithelial surfaces by means of chloride secretion and the inhibition of sodium absorption ${ }^{1,2}$. CFTR dysfunction can directly affect airway immunity by increasing the production of proinflammatory mediators and impairing the immun response to pathogens ${ }^{2,3}$.

Airway inflammation is a central contributor to the pathogenesis of CF pulmonary disease, it may precede bacterial infection and play an independent role in favouring airway dysfunction and damage ${ }^{3}$. Lung disease is the most important problem for majority of patients and has the greatest impact on their quality of life and survival; so limiting the excessive production of inflammatory mediators represents a major therapeutic strategy for slowing the decline in lung function and improving survival ${ }^{2}$. The use of antimicrobial treatment considered to control bacterial infections can reduce the degree of inflammation and therefore the progression to permanent lung damage; so long term treatment with azithromycin (AZM) is included in the current guidelines for CF patients aged $\geq 6$ years ${ }^{4}$.

AZM has microbiological, immunomodulatory and anti-inflammatory properties that can reduce progressive lung damage associated with CF. Although it is not active against Pseudomonas aeruginosa, it can be efficiently used in $P$. aeruginosa infections because sub-inhibitory concentrations can reduce their pathogenic role by interfering with some bacterial activities and increasing their susceptibility to antibiotics ${ }^{5}$. AZM is effective during its first year of administration, but the impact of longer treatment is debated. Also the use of AZM include the possible emergence of antibiotic resistance in the other bacterial pathogens that usually colonise CF patients and the incidence of adverse effects ${ }^{2}$.

Since the first studies of AZM and CF about 15 years ago, a number of clinical trials have investigated the effect of chronic AZM administration on the evaluation of the disease $^{6}$. The first aim of this study was to determine if long term AZM administration improved lung function, nutritional status and decreased pulmonary exacerbations in $\mathrm{CF}$ patients chronically infected with $P$. aeruginosa. Our second aim was to investigate the AZM effect on the incidence of colonization with $S$. aureus and changes in the occurrence in macrolide resistance of this microorganism.

\section{Material and Methods}

\section{Study design and patients}

Twenty four patients with CF aged between 4-23 years followed at Hacettepe University Department of Pediatric Pulmonology between May 2007 and December 2014 were retrospectively recruited the study from $630 \mathrm{CF}$ patients. Ethical approval was received from the Hacettepe University local institutional review board. Inclusion criteria were as follows: a documented diagnosis of CF based on characteristic symptoms and signs, plus a positive sweat test, or two defined CF gene mutations, chronic infection with $P$. aeruginosa and forced expiratory volume in 1 second (FEV1) $>30 \%$ of the predicted value. Chronic infection is defined as more than $50 \%$ of cultures being $P$. aeruginosa positive in the preceding year according to Leeds crtiteria ${ }^{7}$. Criteria were as follows: nontuberculosis mycobacteria (NTM) within 2 years of screening, history of biliary cirrhosis or portal hypertension or liver function results of 2 or more times the upper limit of normal and known allergy or intolerance to macrolides.

After patients had provided written informed consent, their medical histories were reviewed and patients received $10 \mathrm{mg} / \mathrm{kg} /$ day of AZM three times a week. Medical history, oximetry, body mass index (BMI), pulmonary function parameters, sputum/throat cultures, number of total pulmonary exacerbations and number of pulmonary exacerbations that required intravenous antibiotics and hospitalization were analyzed initially and at the visits of months 6 , 9 and 12. Patients were also divided into two groups based on the CFTR mutation classes. Patients with class 1,2 and 3 mutations are called group A; and class 4 and 5 mutations are called group B. The patients continued to taking AZM after the ending period of 12 months. The patients had no other treatment change during their clinical follow-up.

Pulmonary exacerbation was defined as occurring when a patient had any four of the 
following 12 signs or symptoms: change in sputum, new or increased hemoptysis, increased cough, increased dyspnea, malaise fatigue, or lethargy, temperature above $38^{\circ} \mathrm{C}$, anorexia or weight loss, sinus pain or tenderness, change in sinus discharge, change in physical examination of the chest, decrease in pulmonary function by 10 percent or more from a previous value or X-ray changes suggestive of pulmonary infection ${ }^{8}$. Pulmonary exacerbations were evaluated by the number of episodes observed during the follow-up period of each six months. Initial pulmonary exacerbations were defined as number of pulmonary exacerbations for the lifetime before AZM treatment was started.

Pulmonary function testing was performed in accordance with American Thoracic Society standards ${ }^{9}$. The FEV1 measurements were expressed in liters and referenced to a healthy population as a percentage of predicted value.

A standard microbiologic evaluation of sputum and throat cultures for potential pathogens was performed at each clinical visit. Cultures were analyzed for presence of $P$. aeruginosa and resistance against ciprofloxacin, polymyxin, aminoglycoside and $\beta$-lactam antibiotics. At the same time cultures were examined for presence of $S$. aureus and the macrolide (erythromycin and azithromycin and/or clarithromycin) resistance pattern. Acid fast bacillus smear and culture for NTM were performed on sputum specimens yearly.

For monitoring adverse events, reported symptoms, clinical findings and laboratory investigations including complete blood count, liver function and creatinine were recorded.

\section{Statistical analysis}

Data was analyzed using SPSS 20.0. Continuous variables were presented as a mean \pm standard deviation (SD) for normal distribution or median (min-max) for non-normal distribution. Categorical variables were presented as a frequency. Pulmonary function values, BMI, oximetry and number of pulmonary exacerbations were compared with a Repeated Anova test and Tukey post-hoc test if normally distributed and with a Friedman test and Bonferroni adjusted Wilcoxon's test if not normally distributed. Number of patients harbouring specific microorganisms and number of macrolide resistant strains were compared using chi-square test. Statistical tests were two sided and statistical signifiance was accepted at $\mathrm{p}<0.05$.

\section{Results}

In this period, a total of 146 patients were identified as having chronic $P$. aeruginosa colonization and 24 of these patients (13 Male/11 Female) were started on AZM as maintenance therapy. Median age of the patients was 14.7 (range 4-23 years) years. 23 patients had bronchiectasis and only one patient had atelectasis at initial time of AZM treatment. Median FEV1\% was found $68 \%$ (range 30\%-100\%), BMI was found $17.05 \mathrm{~kg} /$ $\mathrm{m}^{2}$ (range 13.3-26.5) and oxygen saturation was found $95 \%$ (range $84 \%-99 \%$ ). Nine patients had co-colonization with $S$. aureus. Before the AZM treatment; median duration of $P$. aeruginosa colonization was 32 months (range 2 months-15 years). The total number of pulmonary exacerbations was 8 (range 1-37) and severe pulmonary exacerbations needing hospitalization was 3 (range 0-7) for the lifetime. Baseline clinical characteristics of the patients were shown in Table I.

Median duration of AZM use was 14 months (range 6-60 months). None of the patients suffered from side effects of AZM. At the end of the 6th, 9th and 12th months of AZM therapy; no significant differences in FEV1\% $(p=0.29)$ and oxygen saturation parameters $(\mathrm{p}=0.70)$ were found compared to the initial time. The median relative change in FEV $1 \%$ predicted was found $7 \%$ at the 12 th month of AZM treatment with nonsignificant improvement. However BMI increased significantly $(p=0.03)$ at the end of the 6th and 12th months of the AZM therapy. Table II and Figure 1 show the changes in FEV1\%, oxygen saturation and BMI during the treatment.

The number of pulmonary exacerbations per patient per year were significantly reduced $(\mathrm{p}<0.001)$ and severe exacerbations $(\mathrm{p}<0.001)$ needing intravenous antibiotic treatment were significantly reduced at the 6 th and 12 th month of AZM treatment (Table II).

At the end of the 12 th month of AZM treatment; $P$. aeruginosa has been eradicated in 1 patient and 14 patients had co-colonization with $S$. aureus and 3 patients had co-colonization with S. aureus and Aspergillus, so Methicillin 
Table I. The Clinical Characteristics of Cystic Fibrosis Patients at Initial Azithromycin Treatment

\begin{tabular}{lcc}
\hline & \multicolumn{2}{c}{ Patients (n=24) } \\
& Mean \pm SD & Median (Range) \\
\hline Initial Age, years & $13.5 \pm 5.4$ & 14.7 (4-23 years) \\
Gender (Male/Female) & $13 / 11$ & \\
CFTR Genotype (n,\%) & & \\
Group A- (Class 1-2-3) & $15(62.5 \%)$ & \\
Group B- (Class 4-5) & $9(37.5 \%)$ & \\
Bronchiectasis (n,\%) & $23(95.8 \%)$ & $95(84-99)$ \\
Oxygen saturation(\%) & $94 \pm 3$ & 68 (30-100) \\
Body mass index (BMI) & $17.4 \pm 3.01$ & \\
FEV1 \% Predicted & $69 \pm 20$ & \\
Co-colonization S. aureus (n,\%) & $9(37.5 \%)$ & \\
Resistant Pseudomonas aeruginosa (n,\%) & $5(20.8 \%)$ & 3 (1-37) \\
Total number of pulmonary exacerbations for lifetime & $9 \pm 8$ & \\
Number of Pulmonary Exacerbations Requiring IV & $3 \pm 2$ & \\
$\quad$ Antibiotic Treatment & & \\
\hline
\end{tabular}

SD: Standard Deviation, FEV1: Forced expiratory volume in 1 second, CFTR: Cystic fibrosis transmembrane conductance regulator

sensitive $S$. aureus (MSSA) colonization was significantly increased $(\mathrm{p}=0.005)$. At the end of the 12th month of AZM; increased $P$. aeruginosa antibiotic resistance to any of $\beta$-lactam or aminoglycoside was detected $(\mathrm{p}=0.01)$. Also macrolide resistance increased $26 \%$ to $63 \%$ respectively in patients with MSSA colonization after AZM treatment $(p=0.008)$. All the patients had negative sputum results for NTM infection during the follow-up period.

\section{Discussion}

The airway inflammatory response in CF is characterised by neutrophilic infiltration and pro-inflammatory cytokine production ${ }^{10,11}$. Accumulation of activated neutrophils with release of toxic products impairs host defence and contributes to chronic infection by microorganisms such as $P$. aeruginosa. The mechanism of macrolides effect on CF lung disease is unknown. The possibility that macrolides might modify the neutrophilic inflammatory response in CF was investigated and their beneficial effects have been confirmed in a number of clinical trials both in adults and children ${ }^{12-18}$.

Although there were differences between the studies, data showed that 6 months of treatment with AZM was effective in improving respiratory function, as shown by the changes in FEV1 and forced vital capacity (FVC). Also, the patients receiving AZM were more likely to be free of exacerbations, at less risk of requiring of oral or intravenous antibiotics, gained more weight and had a better quality of life 7 . Better results were apparently obtained in patients infected by $P$. aeruginosa. In uninfected subjects, AZM only reduced the number of exacerbations and did not improve in lung function, thus suggesting that its beneficial effects were mainly due to its microbiological activity ${ }^{16}$. AZM has no direct bactericidal effect on $P$. aeruginosa, but may reduce the organism virulence by altering biofilm formation, decreasing bacterial adherence to epithelial cells, inhibiting bacterial motility or acting synergistically with other antibiotics ${ }^{19}$.

Although, this study could not to be designed as a placebo controlled study, the results showed that at least 6 months of AZM treatment led to a significiant reduction in the number of pulmonary exacerbations requiring antibiotics. The reduced number of pulmonary exacerbations requiring courses of antibiotics documented here may be related with the anti-inflammatory properties of AZM. Also it is unclear whether the clinical benefits may be maintained in the longer term, beyond 12 months. Two studies have evaluated the impact 
Table II. Alterations in Clinical Characteristics Following to Azithromycin Treatment

\begin{tabular}{lccccc}
\hline & $\begin{array}{c}\text { Month 0 } \\
\text { Median (Range) }\end{array}$ & $\begin{array}{c}\text { Month 6 } \\
\text { Median (Range) }\end{array}$ & $\begin{array}{c}\text { Month 9 } \\
\text { Median (Range) }\end{array}$ & $\begin{array}{c}\text { Month 12 } \\
\text { Median (Range) }\end{array}$ & P value \\
\hline $\begin{array}{l}\text { FEV1 (\%) } \\
\begin{array}{l}\text { Oxygen } \\
\text { Saturation (\%) }\end{array}\end{array}$ & $68(30-100)$ & $79(27-119)$ & $79(47-114)$ & $75(46-104)$ & 0.29 \\
$\begin{array}{l}\text { Body mass } \\
\text { index (BMI) }\end{array}$ & $17.05(13.3-26.5)^{\mathrm{a}}$ & $18.3(13.6-26.7)^{\mathrm{b}}$ & $18.9(14.2-27.1)^{\mathrm{b}}$ & $18.5(14.5-26)^{\mathrm{b}}$ & 0.03 \\
$\begin{array}{l}\text { Total Number } \\
\text { of Pulmonary }\end{array}$ & $8(1-37)^{\mathrm{a}}$ & $1(0-3)^{\mathrm{b}}$ & $0(0-1)^{\mathrm{b}}$ & $1(0-2)^{\mathrm{b}}$ & $\mathrm{P}<0.001$ \\
$\begin{array}{l}\text { Exacerbations } \\
\text { Number of }\end{array}$ & $3(0-7)^{\mathrm{a}}$ & $0(0-1)^{\mathrm{b}}$ & $0(0-1)^{\mathrm{b}}$ & $0(0-1)^{\mathrm{b}}$ & $\mathrm{P}<0.001$ \\
$\begin{array}{l}\text { Pulmonary } \\
\text { Exacerbations }\end{array}$ & & & & & \\
$\begin{array}{l}\text { Requiring IV } \\
\text { Antibiotic } \\
\text { Treatment }\end{array}$ & & & & & \\
\hline
\end{tabular}

a $\mathrm{p}<0.05$ : Time of month 0 versus time of month 6, 9 and 12

${ }^{b} \mathrm{p}>0.05$ : Time of month 6, 9 and 12

FEV1: Forced expiratory volume in 1 second, IV: Intravenous

of longer periods of treatment, and both found that efficacy was poor after the first year ${ }^{20,21}$. Tramper-Stranders et al. ${ }^{20}$ found that AZM administered for 3 years had a positive effect on the percentage of predicted FEV1 in the first year, but this percentage subsequently returned to pretreatment levels. Similarly, Willekens et $a .^{21}$ found that although there was no change in pulmonary function parameters or the incidence of severe exacerbations requiring intravenous antibiotic treatment, the first year of AZM administration was associated with a significant reduction in the number of orally treated pulmonary exacerbations per patient. However, this reduction was not maintained in the second and third treatment years. The authors therefore concluded that AZM therapy should be limited to 6-12 months and after this period the risk of drug-related problems could outweigh the advantages ${ }^{21}$.

In this study, we did not find significant improvement in the FEV1 from baseline to the 6 th and 12th month of treatment. Possible explanation for analysis of the FEV1 results in our population may be related with the large variation in individual FEV1 measurements during the study. A meta-analysis of studies in patients with CF who predominantly had chronic infection with $P$. aeruginosa established that AZM treatment improved FEV1 by $3.2 \% 22$. However Clement et al. ${ }^{15}$ found no difference (probably because of early stages of lung
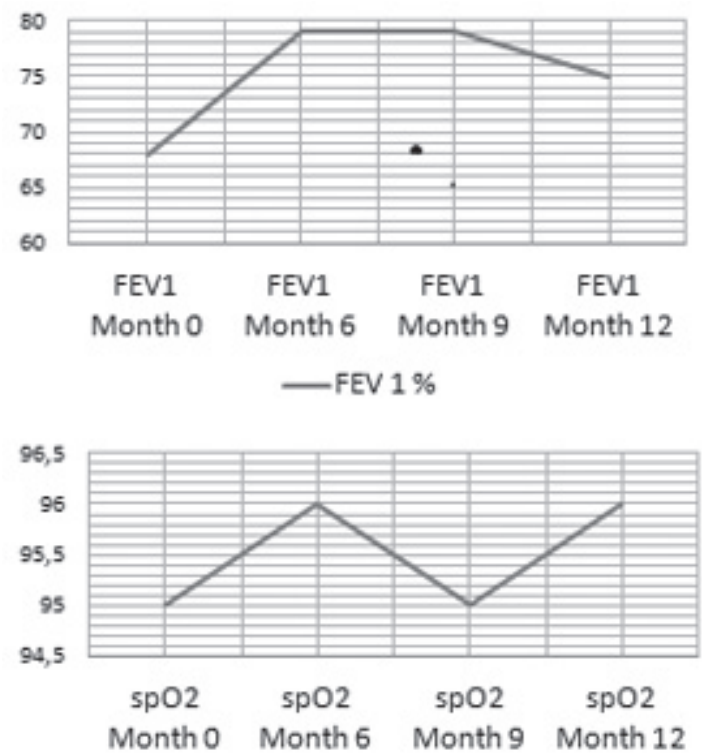

$-5 \mathrm{pO} 2 \%$

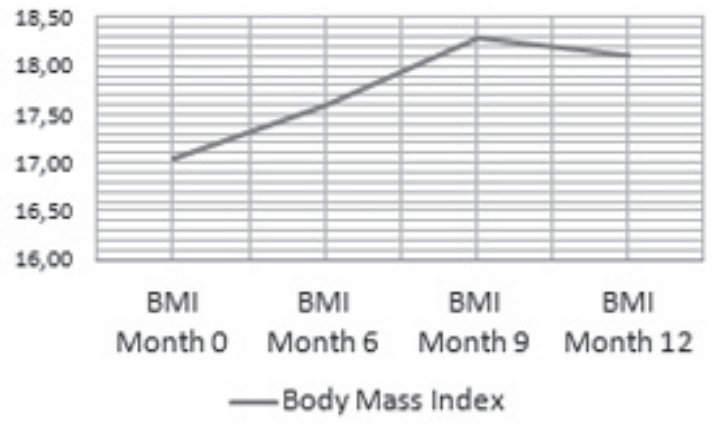

Fig. 1. Changes in FEV1\%, oxygen saturation and body mass index (BMI) during the azithromycin treatment 
disease in the population studied) in FEV1 with AZM treatment. Saimon et al. ${ }^{17}$ also showed that participants treated with AZM in whom the pulmonary function did not improve still experienced clinical benefits with a reduction in the number of pulmonary exacerbations. Even patients who did not experience an improvement in pulmonary function from AZM treatment which share similarities with our present data; they experienced clinical benefits reflected by reduction in the number of acute pulmonary exacerbations.

Malnutrition and growth retardation are frequently observed in patients with $\mathrm{CF}$ and there is not enough information about the effect of AZM treatment on nutritional status. The impact of long-term AZM treatment on the weight changes was assessed in all four studies $12,14,15,21$. Saiman et al. ${ }^{14}$ noted that the participants in the AZM arm had a higher rate of weight gain than the placebo group by the end of the trial. The other two studies reported no statistically significant differences in BMI between AZM and placebo groups, with a similar trend in patients infected with P. aeruginosa ${ }^{12,15}$. Willekens et al. $^{21}$ also found no significant difference in BMI at the end of the first treatment year compared to the year before treatment start. In this study we found significant improvement on nutritional status after AZM treatment. It may be related with the wide range in BMI of the patients.

The use of AZM can also lead to the emergence of macrolide resistance among other significant CF pathogens ${ }^{22}$. Hansen et al. ${ }^{23}$ studied 70 CF patients on long term AZM for at least 3 months. Although AZM treatment reduced the prevalence of $S$. aureus, $H$. influenzae and $S$. pneumoniae, it led to an increase of macrolide resistance in $S$. aureus isolates from these patients. Similarly, Tramper-Stranders et al. ${ }^{20}$ studied 100 patients with a mean age at initiation of therapy of 10.8 years who received AZM for a mean duration of 3.5 years. They found that macrolide resistance among patients colonized with $S$. aureus rose from $10 \%$ before initiation of therapy to $83 \%$, $97 \%$ and $100 \%$ after one, two and three years of therapy respectively. On the other hand, $S$. aureus colonization decreased slightly but not significantly after AZM therapy, from $50 \%$ initially to $48 \%, 44 \%$ and $36 \%$ in the following three years ${ }^{17,20}$. In our report, we also found increased macrolide resistance of $S$. aureus after the treatment, however $S$. aureus isolation rate has increased among the patients, different from the other studies.

None of the studies found a significant change in the rate of emergence or eradication of $P$. aeruginosa from the sputum after AZM treatment ${ }^{14,15}$. In our study, $P$. aeruginosa could not been eradicated and at the end of the treatment; increased $P$. aeruginosa antibiotic resistance to $\beta$-lactam and aminoglycoside has been detected. Tramper-Standers et al. ${ }^{20}$ also found increased $P$. aeruginosa antibiotic resistance in their study at the end of AZM treatment. However due to the lack of a placebo group, $P$. aeruginosa antibiotic resistance may be related with a result of repeated exposure to antibiotics for pulmonary exacerbations.

Because this was a retrospective review, there is no blinding and lack of control group, the results preclude definitive statements regarding the effectiveness of AZM in CF patients. Also the other limitation of this study is that it is from a single center with a small group of CF patients.

Finally, there is some concern that the chronic use of AZM in subjects with NTM infection can lead to resistance thus complicating NTM treatment ${ }^{14,24}$. In our study we also checked NTM in culture every year and had negative results for NTM infection.

In conclusion; this study is the first look at AZM treatment in Turkish CF patients who have a different distribution of genotypes. Despite increased $P$. aeruginosa antibiotic resistance and MSSA colonization rates, the lower incidence of acute exacerbations in patients receiving AZM is an important and clinically relevant measure of beneficial effect. Although this is a single center and small population of study, long term use of AZM may be considered to slow pulmonary deterioration in CF patients with $P$. aeruginosa colonization. Also the emergence of resistance to macrolide antibiotics in oral flora, potentially S. aureus, and NTM should be monitored during long term therapy. 


\section{REFERENCES}

1. Davies JC, Alton EW, Bush A. Cystic fibrosis. BMJ 2007; 335: 1255-1259.

2. Principi N, Blasi F, Esposito S. Azithromycin use in patients with cystic fibrosis. Eur J Clin Microbiol Infect Dis 2015; 34: 1071-1079.

3. Watt AP, Courtney J, Moore J, Ennis M, Elborn JS. Neutrophil cell death, activation and bacterial infection in cystic fibrosis. Thorax 2005; 60: 659-664.

4. Mogayzel PJ Jr, Naureckas ET, Robinson KA, et al. Cystic fibrosis pulmonary guidelines. Chronic medications for maintenance of lung health. Am J Respir Crit Care Med 2013; 187: 680-689.

5. Lutz L, Pereira DC, Paiva RM, et al. Macrolides decrease the minimal inhibitory concentration of antipseudomonal agents against Pseudomonas aeruginosa from cystic fibrosis patients in biofilm. BMC Microbiol 2012; 12: 196.

6. Southern KW, Barker PM, Solis-Moya A, et al. Macrolide antibiotics for cystic fibrosis. Cochrane Database Syst Rev 2012; 11: CD002203.

7. Lee TW, Brownlee KG, Conway SP, et al. Evaluation of a new definition for chronic Pseudomonas aeruginosa infection in cystic fibrosis patients. J Cyst Fibros 2003; 2: $29-34$

8. Goss $\mathrm{CH}$, Burns JL. Exacerbations in cystic fibrosis. 1: epidemiology and pathogenesis. Thorax 2007; 62: 360-367.

9. Standardization of Spirometry, 1994 Update. American Thoracic Society. Am J Respir Crit Care Med 1995; 152: 1107-1136.

10. Khan TZ, Wagener JS, Bost T, et al. Early pulmonary inflammation in infants with cystic fibrosis. Am J Respir Crit Care Med 1995; 151: 1075-1082.

11. Spagnolo P, Fabbri LM, Bush A. Long-term macrolide treatment for chronic respiratory disease. Eur Respir J 2013; 42: 239-251.

12. Wolter J, Seeney S, Bell S, et al. Effect of long term treatment with azithromycin on disease parameters in cystic fibrosis: a randomized trial. Thorax 2002; 57: 212-216.

13. Equi A, Balfour-Lynn IM, Bush A, et al. Long term azithromycin in children with cystic fibrosis: a randomized, placebo-controlled crossover trial. Lancet 2002; 360: 978-984.
14. Saiman L, Marshall BC, Mayer-Hamblett N, et al. Azithromycin in patients with cystic fibrosis chronically infected with Pseudomonas aeruginosa: a randomized controlled trial. JAMA 2003; 290: 1749-1756.

15. Clement A, Tamalet A, Leroux E, et al. Long term effects of azithromycin in patients with cystic fibrosis: a double blind, placebo controlled trial. Thorax 2006; 61: 895-902.

16. McCormack J, Bell S, Senini S, et al. Daily versus weekly azithromycin in cystic fibrosis patients. Eur Respir J 2007; 30: 487-495.

17. Saiman L, Anstead M, Mayer-Hamblett N, et al. Effect of azithromycin on pulmonary function in patients with cystic fibrosis uninfected with Pseudomonas aeruginosa: a randomized controlled trial. JAMA 2010; 303: $1707-1715$.

18. Saiman L, Mayer-Hamblett N, Anstead M, et al. Openlabel, follow-on study of azithromycin in paediatric patients with CF uninfected with Pseudomonas aeruginosa. Pediatr Pulmonol 2012; 47: 641-648.

19. Ratjen F, Saiman L, Mayer-Hamblett N, et al. Effect of azithromycin on systemic markers of inflammation in cystic fibrosis patients uninfected with Pseudomonas aeruginosa. Chest 2012; 142: 1259-1266.

20. Tramper-Stranders GA, Wolfs TF, et al. Maintenance azithromycin treatment in pediatric patients with cystic fibrosis: long-term outcomes related to macrolide resistance and pulmonary function. Pediatr Infect Dis J 2007; 26: 8-12.

21. Willekens J, Eyns H, Malfroot A. How long should we maintain long-term azithromycin treatment in cystic fibrosis patients? Pediatr Pulmonol 2015; 50: 103-104.

22. Cai Y, Chai D, Wang R, et al. Effectiveness and safety of macrolides in cystic fibrosis patients: a meta-analysis and systematic review. J Antimicrob Chemother 2011; 66: 968-978.

23. Hansen CR, Pressler T, Koch C, et al. Long-term azitromycin treatment of cystic fibrosis patients with chronic Pseudomonas aeruginosa infection; an observational cohort study. J Cyst Fibros 2005; 4: 35-40.

24. Roux AL, Catherinot E, Ripoll F, et al. Multicenter study of prevalence of nontuberculous mycobacteria in patients with cystic fibrosis in france. J Clin Microbiol 2009; 47: 4124-4128 